\title{
On functionals involving the torsional rigidity related to some classes of nonlinear operators
}

\author{
Francesco Della Pietra, Nunzia Gavitone, Serena Guarino Lo Bianco \\ Università degli studi di Napoli Federico II, Dipartimento di Matematica e Applicazioni "R. Caccioppoli" \\ Via Cintia, Monte S. Angelo - 80126 Napoli, Italia.*
}

September 19, 2018

\begin{abstract}
In this paper we study optimal estimates for two functionals involving the anisotropic $p$-torsional rigidity $\mathrm{T}_{p}(\Omega), 1<p<+\infty$. More precisely, we study $\Phi(\Omega)=\frac{\mathrm{T}_{\mathrm{p}}(\Omega)}{|\Omega| M(\Omega)}$ and $\Psi(\Omega)=\frac{\mathrm{T}_{\mathrm{p}}(\Omega)}{|\Omega|\left[\mathrm{R}_{\mathrm{F}}(\Omega)\right]^{\mathrm{p}-1}}$, where $\mathrm{M}(\Omega)$ is the maximum of the torsion function $u_{\Omega}$ and $R_{F}(\Omega)$ is the anisotropic inradius of $\Omega$.
\end{abstract}

KEYWORDS: torsional rigidity, anisotropic operators, optimal estimates

MSC 2010: 49Q10, 35J25

Let $F: \mathbb{R}^{N} \rightarrow\left[0,+\infty\left[, N \geqslant 2\right.\right.$, be a convex, even, 1-homogeneous and $C^{3, \beta}\left(\mathbb{R}^{N} \backslash\{0\}\right)$ function such that $\left[\mathrm{FP}^{\mathrm{P}}\right]_{\xi \xi}$ is positive definite in $\mathbb{R}^{\mathrm{N}} \backslash\{0\}, 1<\mathrm{p}<+\infty$. The anisotropic $p$-laplacian is the operator defined by

$$
\mathcal{Q}_{p} u:=\sum_{i=1}^{N} \frac{\partial}{\partial x_{i}}\left(F(\nabla u)^{p-1} F_{\xi_{i}}(\nabla u)\right) .
$$

For $p=2, Q_{2}$ is the so-called Finsler Laplacian, while when $F(\xi)=|\xi|$ is the Euclidean norm, $Q_{p}$ reduces to the well known $p$-Laplace operator.

Given a bounded domain $\Omega$ in $\mathbb{R}^{\mathrm{N}}$, let us consider the torsion problem for $Q_{\mathrm{p}}$ :

$$
\begin{cases}-Q_{\mathfrak{p}} u=1 & \text { in } \Omega \\ u=0 & \text { on } \partial \Omega\end{cases}
$$

The anisotropic $p$-torsional rigidity of $\Omega$ is the number $T_{p}(\Omega)>0$ defined by

$$
\mathrm{T}_{\mathrm{p}}(\Omega)=\int_{\Omega} \mathrm{F}\left(\nabla \mathrm{u}_{\Omega}\right)^{\mathrm{p}} \mathrm{dx}=\int_{\Omega} \mathrm{u}_{\Omega} \mathrm{dx}
$$

where $u_{\Omega} \in W_{0}^{1, p}(\Omega)$ is the torsion function, that is the unique solution of (1).

\footnotetext{
*Email: f.dellapietra@unina.it, nunzia.gavitone@unina.it, serena.guarinolobianco@unina.it
} 
The main aim of the paper is the study of optimal estimates for the following two functionals involving $T_{p}(\Omega)$ :

$$
\Phi(\Omega)=\frac{\mathrm{T}_{\mathrm{p}}(\Omega)}{|\Omega| \mathrm{M}(\Omega)}, \quad \Psi(\Omega)=\frac{\mathrm{T}_{\mathrm{p}}(\Omega)}{|\Omega|\left[\mathrm{R}_{\mathrm{F}}(\Omega)\right]^{\mathrm{q}}} .
$$

Here and after we will denote by $q$ the Hölder conjugate of $p, q=\frac{p}{p-1}$, by $M(\Omega)$ the maximum of the torsion function $u_{\Omega}$ and by $\operatorname{R}_{F}(\Omega)$ the anisotropic inradius of $\Omega$ (see Section 2 for the precise definitions). Observe that the functionals $\Phi$ and $\Psi$ are scaling invariant with respect to the domain. Indeed:

$$
\mathrm{T}_{\mathrm{p}}(\mathrm{t} \Omega)=\mathrm{t}^{\mathrm{N}+\mathrm{q}} \mathrm{T}_{\mathrm{p}}(\Omega), \quad|\mathrm{t} \Omega|=\mathrm{t}^{\mathrm{N}}|\Omega|, \quad \mathrm{M}(\mathrm{t} \Omega)=\mathrm{t}^{\mathrm{q}} \mathrm{M}(\Omega), \quad \mathrm{R}_{\mathrm{F}}(\mathrm{t} \Omega)=\mathrm{tR}_{\mathrm{F}}(\Omega) .
$$

Our main result is the following.

Theorem 1.1. Let $\Omega$ be a convex bounded domain in $\mathbb{R}^{\mathrm{N}}$. It holds that

i) $\frac{\mathrm{q}}{\mathrm{N}^{-1}(\mathrm{~N}+\mathrm{q})} \leqslant \Phi(\Omega) \leqslant \frac{\mathrm{q}}{\mathrm{q}+1}$.

The right-hand side inequality is optimal for a suitable sequence of thinning rectangles.

ii) $\frac{1}{\mathrm{Nq}-1} \frac{1}{\mathrm{~N}+\mathrm{q}} \leqslant \Psi(\Omega) \leqslant \frac{1}{\mathrm{q}+1}$.

The left-hand side inequality holds as an equality if and only if $\Omega$ is a Wulff shape, that is a ball in the dual norm $\mathrm{F}^{\mathrm{o}}$; the right-hand side inequality is optimal for a suitable sequence of thinning rectangles.

When $F=\mathcal{E}$ is the Euclidean norm, there is a wide literature on sharp estimates for $T_{p}(\Omega)$ related to several geometrical quantities depending on $\Omega$. For example, in the classical case of the torsional rigidity for the Laplace operator $(p=2)$, with $N=2$, it is known that

$$
\frac{1}{8} \leqslant \Psi(\Omega)=\frac{T_{2}(\Omega)}{R_{\varepsilon}^{2}|\Omega|} \leqslant \frac{1}{3},
$$

where $R_{\mathcal{E}}(\Omega)$ is the standard Euclidean inradius of $\Omega$. The left-hand side inequality is due to Pólya and Szegő (see [PZ]), while the right-hand side inequality was proved by Makai in [M]. As regards the case $p \neq 2$, in [FGL], among other results, estimates for $\Psi(\Omega)$ are given in the planar case, obtaining an upper bound and a sharp lower bound. In the anisotropic case, in [BGM] the estimates in $i$ ) are proved for $p=2$.

As regards the functional $\Phi(\Omega)$, up to our knowledge, it seems that the only known result is in the Euclidean case for $p=2$. Indeed, in [HLP] the authors prove the following estimates:

$$
\frac{1}{(\mathrm{~N}+1)^{2}} \leqslant \Phi(\Omega)=\frac{\mathrm{T}_{2}(\Omega)}{|\Omega| \mathrm{M}(\Omega)} \leqslant \frac{2}{3} .
$$

Moreover, they show the optimality of the upper bound, while they conjecture that the lower bound is not optimal, and that the sharp constant in the plane is $\frac{1}{3}$, achieved on a sequence of thinning isosceles triangles. In our result, we improve the constant $(N+1)^{-2}$, replacing it with $[\mathrm{N}(\mathrm{N}+2)]^{-1}$. Anyway, we believe that $[\mathrm{N}(\mathrm{N}+2)]^{-1}$ is not optimal, and for $\mathrm{N}=2$ we show that there is a sequence of thinning isosceles triangles $\tau_{n}$ such that $\Phi\left(\tau_{n}\right) \rightarrow \frac{1}{3}$.

In order to prove our main result, among the main tools involved, the following estimate for the maximum $M(\Omega)$ of the torsion function $u_{\Omega}$ plays a key role. 
Theorem 1.2. Let $\Omega$ be a bounded convex domain in $\mathbb{R}^{N}, N \geqslant 2$, and let $R_{\mathrm{F}}(\Omega)$ the anisotropic inradius of $\Omega$. Let $\mathfrak{u}_{\Omega}$ be the solution of (1). For $1<p<+\infty$ it holds that

$$
\frac{\mathrm{R}_{\mathrm{F}}^{\mathrm{q}}(\Omega)}{\mathrm{qNq}-1} \leqslant M(\Omega) \leqslant \frac{\mathrm{R}_{\mathrm{F}}^{\mathrm{q}}(\Omega)}{\mathrm{q}} .
$$

The right-hand side inequality is optimal for a suitable sequence of thinning $\mathrm{N}$-rectangular domains. The other inequality, holds as an equality if and only if $\Omega$ is the Wulff shape $\mathcal{W}_{R}\left(x_{0}\right)$.

The upper bound in (2) has been proved in $[P]$ in the Euclidean case for $p=2, N=2$ (see also [S]), by using a $\mathcal{P}$-function computation and a maximum principle. Anyway, many other estimates for the torsion function are known; the interested reader can refer, for example, to $[\mathrm{vB}, \mathrm{BFr}, \mathrm{HLP}]$ and the reference therein contained. We prove inequality (2) generalizing the $\mathcal{P}$-function technique to the case $1<p<+\infty$, and in the anisotropic case.

Finally, we recall that in the Euclidean case, several other estimates for the p-torsional rigidity, involving different geometrical quantities, are known (for the Eucidean case, see for instance [vBBV, vBFNT, S] $(p=2),[F G L](1<p<+\infty)$, and [DG1] for the anisotropic case $(1<p<+\infty))$.

The paper is organized as follows. In Section 2 we fix some notation and recall prelimiary results about Finsler metrics and the anisotropic p-torsional rigidity. In Section 3 we prove Theorem 1.2 by using the $\mathcal{P}$-function method. Finally, in Section 4 we give the proof of the main Theorem 1.1. We will split it in several partial results.

\section{NOTATION AND PRELIMINARIES}

Throughout the paper we will consider a convex even 1-homogeneous function

$$
\xi \in \mathbb{R}^{\mathrm{N}} \mapsto \mathrm{F}(\xi) \in[0,+\infty[,
$$

that is a convex function such that

$$
F(t \xi)=|t| F(\xi), \quad t \in \mathbb{R}, \xi \in \mathbb{R}^{N},
$$

and such that

$$
a|\xi| \leqslant F(\xi), \quad \xi \in \mathbb{R}^{N},
$$

for some constant $a>0$. The hypotheses on F imply there exists $b \geqslant a$ such that

$$
\mathrm{F}(\xi) \leqslant \mathrm{b}|\xi|, \quad \xi \in \mathbb{R}^{\mathrm{N}} .
$$

Moreover, throughout the paper we will assume that $F \in C^{3, \beta}\left(\mathbb{R}^{N} \backslash\{0\}\right)$, and

$$
\left[\mathrm{F}^{\mathrm{p}}\right]_{\xi \xi}(\xi) \text { is positive definite in } \mathbb{R}^{\mathrm{N}} \backslash\{0\},
$$

with $1<p<+\infty$.

The hypothesis (6) on $\mathrm{F}$ ensures that the operator

$$
Q_{\mathrm{p}} \mathrm{u}:=\operatorname{div}\left(\frac{1}{\mathrm{p}} \nabla_{\xi}\left[\mathrm{F}^{\mathrm{p}}\right](\nabla \mathrm{u})\right)
$$

is elliptic, hence there exists a positive constant $\gamma$ such that

$$
\frac{1}{p} \sum_{i, j=1}^{n} \nabla_{\xi_{i} \xi_{j}}^{2}\left[F^{p}\right](\eta) \xi_{i} \xi_{j} \geqslant \gamma|\eta|^{p-2}|\xi|^{2},
$$

for some positive constant $\gamma$, for any $\eta \in \mathbb{R}^{n} \backslash\{0\}$ and for any $\xi \in \mathbb{R}^{n}$. 
Remark 2.1. We stress that for $p \geqslant 2$ the condition

$\nabla_{\xi}^{2}\left[\mathrm{~F}^{2}\right](\xi)$ is positive definite in $\mathbb{R}^{\mathrm{N}} \backslash\{0\}$,

implies (6).

The polar function $\mathrm{F}^{\mathrm{o}}: \mathbb{R}^{\mathrm{N}} \rightarrow[0,+\infty[$ of $\mathrm{F}$ is defined as

$$
F^{o}(v)=\sup _{\xi \neq 0} \frac{\langle\xi, v\rangle}{F(\xi)}
$$

It is easy to verify that also $F^{O}$ is a convex function which satisfies properties (3) and (4). Furthermore,

$$
F(v)=\sup _{\xi \neq 0} \frac{\langle\xi, v\rangle}{F^{o}(\xi)} .
$$

From the above property it holds that

$$
|\langle\xi, \eta\rangle| \leqslant F(\xi) F^{o}(\eta), \quad \forall \xi, \eta \in \mathbb{R}^{N} .
$$

The set

$$
\mathcal{W}=\left\{\xi \in \mathbb{R}^{\mathrm{N}}: \mathrm{F}^{\mathrm{o}}(\xi)<1\right\}
$$

is the so-called Wulff shape centered at the origin. We put $\kappa_{n}=|\mathcal{W}|$, where $|\mathcal{W}|$ denotes the Lebesgue measure of $\mathcal{W}$. More generally, we denote with $\mathcal{W}_{r}\left(x_{0}\right)$ the set $r \mathcal{W}+x_{0}$, that is the Wulff shape centered at $x_{0}$ with measure $\kappa_{n} r^{n}$, and $\mathcal{W}_{r}(0)=\mathcal{W}_{r}$.

We observe that $F$ is the support function of $\overline{\mathcal{W}}$. In general for a nonempty closed convex set $K \subset \mathbb{R}^{N}$, the support function $h_{K}$ is defined by

$$
h_{K}(x):=\sup \{\langle x, \xi\rangle, \xi \in K\}, \quad \text { for } x \in \mathbb{R}^{N} \text {. }
$$

The following properties of $F$ and $F^{\circ}$ hold true:

$$
\begin{aligned}
& \left\langle F_{\xi}(\xi), \xi\right\rangle=F(\xi), \quad\left\langle F_{\xi}^{o}(\xi), \xi\right\rangle=F^{o}(\xi), \quad \forall \xi \in \mathbb{R}^{N} \backslash\{0\} \\
& F\left(F_{\xi}^{o}(\xi)\right)=F^{o}\left(F_{\xi}(\xi)\right)=1, \quad \forall \xi \in \mathbb{R}^{N} \backslash\{0\}, \\
& F^{o}(\xi) F_{\xi}\left(F_{\xi}^{o}(\xi)\right)=F(\xi) F_{\xi}^{o}\left(F_{\xi}(\xi)\right)=\xi \quad \forall \xi \in \mathbb{R}^{N} \backslash\{0\} .
\end{aligned}
$$

\subsection{Anisotropic mean curvature}

Let $\Omega$ be a $C^{2}$ bounded domain, and $v(x)$ be the unit outer normal at $x \in \partial \Omega$, and let $u \in C^{2}(\bar{\Omega})$ such that $\Omega_{t}=\{u>t\}, \partial \Omega_{t}=\{u=t\}$ and $\nabla u \neq 0$ on $\partial \Omega_{t}$. The anisotropic outer normal $n_{F}$ to $\partial \Omega_{t}$ is given by

$$
n_{F}(x)=F_{\xi}(v(x))=F_{\xi}(-\nabla u), \quad x \in \partial \Omega .
$$

It holds

$$
\mathrm{F}^{\mathrm{o}}\left(\mathrm{n}_{\mathrm{F}}\right)=1
$$

The anisotropic mean curvature of $\partial \Omega_{t}$ is defined as

$$
\mathcal{H}_{\mathrm{F}}(x)=\operatorname{div}\left(n_{\mathrm{F}}(x)\right)=\operatorname{div}\left[\nabla_{\xi} F(-\nabla u(x))\right], \quad x \in \partial \Omega_{\mathrm{t}} .
$$


It holds that

$$
\frac{\partial u}{\partial n_{\mathrm{F}}}=\nabla u \cdot F_{\xi}(-\nabla u)=-F(\nabla u) .
$$

In $[X]$ it has been proved that for a smooth function $u$, on its level sets $\{u=t\}$ it holds

$$
Q_{2} u=\frac{\partial u}{\partial n_{F}} \mathcal{H}_{F}+\frac{\partial^{2} u}{\partial n_{F}^{2}}
$$

where $\frac{\partial u}{\partial n_{\mathrm{F}}}=\nabla u \cdot n_{\mathrm{F}}$. In the next result we generalize (14) for $\mathcal{Q}_{\mathrm{p}} u$.

Proposition 2.2. Let $\mathrm{u}$ be a $\mathrm{C}^{2}(\bar{\Omega})$ function with a regular level set $\partial \Omega_{\mathrm{t}}$. Then we have

$$
\mathcal{Q}_{\mathrm{p}} \mathrm{u}=\mathrm{F}^{\mathrm{p}-2}(\nabla \mathrm{u})\left(\frac{\partial \mathrm{u}}{\partial \mathrm{n}_{\mathrm{F}}} \mathcal{H}_{\mathrm{F}}+(\mathrm{p}-1) \frac{\partial^{2} \mathrm{u}}{\partial \mathrm{n}_{\mathrm{F}}^{2}}\right),
$$

where $\mathcal{H}_{\mathrm{F}}$ is the anisotropic mean curvature of $\partial \Omega_{\mathrm{t}}$ as defined in (12).

Proof. By definition of $Q_{p},(14)$ and (13), we have

$$
\begin{aligned}
\mathcal{Q}_{\mathfrak{p}} u & =\operatorname{div}\left(F^{p-2}(\nabla u) F(\nabla u) F_{\xi}(\nabla u)\right) \\
& =F^{p-2}(\nabla u)\left(Q_{2} u+(p-2) F_{\xi_{i}}(\nabla u) F_{\xi_{j}}(\nabla u) u_{x_{i} x_{j}}\right) \\
& =F^{p-2}(\nabla u)\left(\frac{\partial u}{\partial n_{F}} \mathcal{H}_{F}+\frac{\partial^{2} u}{\partial n_{F}^{2}}+(p-2) F_{\xi_{i}}(\nabla u) F_{\xi_{j}}(\nabla u) u_{x_{i} x_{j}}\right) \\
& =F^{p-2}(\nabla u)\left(\frac{\partial u}{\partial n_{F}} \mathcal{H}_{F}+(p-1) \frac{\partial^{2} u}{\partial n_{F}^{2}}\right)
\end{aligned}
$$

that is the thesis.

Finally we recall the definition of the anisotropic distance from the boundary and the anisotropic inradius.

Let us consider a domain $\Omega$, that is a connected open set of $\mathbb{R}^{N}$, with non-empty boundary.

The anisotropic distance of $x \in \bar{\Omega}$ to the boundary of $\partial \Omega$ is the function

$$
d_{F}(x)=\inf _{y \in \partial \Omega} F^{o}(x-y), \quad x \in \bar{\Omega} .
$$

We stress that when $F=|\cdot|$ then $d_{F}=d_{\varepsilon}$, the Euclidean distance function from the boundary.

It is not difficult to prove that $d_{F}$ is a uniform Lipschitz function in $\bar{\Omega}$ and, using the property of $F$ we have

$$
\mathrm{F}\left(\nabla \mathrm{d}_{\mathrm{F}}(\mathrm{x})\right)=1 \text { a.e. in } \Omega \text {. }
$$

Obviously, assuming $\sup _{\Omega} d_{F}<+\infty, d_{F} \in W_{0}^{1, \infty}(\Omega)$ and the quantity

$$
R_{F}(\Omega)=\sup \left\{d_{F}(x), x \in \Omega\right\},
$$

is called anisotropic inradius of $\Omega$.

For further properties of the anisotropic distance function we refer the reader to [CM]. 


\subsection{Anisotropic p-torsional rigidity}

In this subsection we summarize some properties of the anisotropic p-torsional rigidity. We refer the reader to [DGI] for further details.

Let $\Omega$ be a bounded domain in $\mathbb{R}^{N}$, and $1<p<+\infty$. Throughout the paper we will denote by $q$ the Hölder conjugate of $p$,

$$
q:=\frac{p}{p-1}
$$

Let us consider the torsion problem for the anisotropic $p$-Laplacian

$$
\begin{cases}-Q_{\mathfrak{p}} u:=-\operatorname{div}\left(F^{p-1}(\nabla \mathfrak{u}) F_{\xi}(\nabla \mathfrak{u})\right)=1 & \text { in } \Omega \\ \mathfrak{u}=0 & \text { on } \partial \Omega .\end{cases}
$$

By classical result there exists a unique solution of $(17)$, that we will always denote by $\mathfrak{u}_{\Omega}$, which is positive in $\Omega$. Moreover, by (6) and being $F \in C^{3}\left(\mathbb{R}^{n} \backslash\{0\}\right)$, then $u_{\Omega} \in C^{1, \alpha}(\Omega) \cap$ $\mathrm{C}^{3}\left(\left\{\nabla \mathrm{u}_{\Omega} \neq 0\right\}\right)$ (see [LU, To]).

In view of the above considerations, we define the p-torsional anisotropic rigidity of $\Omega$ the number $T_{p}(\Omega)>0$ such that

$$
\mathrm{T}_{\mathrm{p}}(\Omega)=\int_{\Omega} \mathrm{F}\left(\nabla \mathrm{u}_{\Omega}\right)^{\mathrm{p}} \mathrm{dx}=\int_{\Omega} \mathrm{u}_{\Omega} \mathrm{dx} .
$$

A characterization of $T_{p}$ is provided by the equality $T_{p}(\Omega)=\sigma(\Omega)^{\frac{1}{p-1}}$, where $\sigma(\Omega)$ is the best constant in the Sobolev inequality

$$
\|\varphi\|_{\mathrm{L}^{1}(\Omega)}^{\mathrm{p}} \leqslant \sigma(\Omega)\|\mathrm{F}(\nabla \varphi)\|_{\mathrm{L}^{\mathrm{p}}(\Omega)}^{\mathrm{p}},
$$

that is

$$
\mathrm{T}_{\mathrm{p}}(\Omega)^{\mathrm{p}-1}=\sigma(\Omega)=\max _{\psi \in W_{0}^{1, p}(\Omega) \backslash\{0\}} \frac{\left(\int_{\Omega}|\psi| \mathrm{d} x\right)^{\mathrm{p}}}{\int_{\Omega} \mathrm{F}(\nabla \psi)^{\mathrm{p}} \mathrm{dx}},
$$

and the solution $\mathfrak{u}_{\Omega}$ of (17) realizes the maximum in (19).

It is immediate to see that if $\Omega \subset \tilde{\Omega}$, then

$$
\mathrm{T}_{\mathrm{p}}(\Omega) \leqslant \mathrm{T}_{\mathrm{p}}(\tilde{\Omega}) .
$$

Moreover, by the maximum principle it holds that

$$
M(\Omega) \leqslant M(\tilde{\Omega}),
$$

where $M(\Omega)$ is the maximum of the torsion function in $\Omega$.

A consequence of the anisotropic Pólya-Szegó inequality (see [AFLT]) is the following upper bound for $T_{p}(\Omega)$ in terms of the measure of $\Omega$.

Theorem 2.3. Let $\Omega$ be a bounded open set of $\mathbb{R}^{\mathrm{N}}$. Then,

$$
\mathrm{T}_{\mathrm{p}}(\Omega) \leqslant \mathrm{T}_{\mathrm{p}}\left(\mathcal{W}_{\mathrm{R}}\right),
$$

where $\mathcal{W}_{\mathrm{R}}$ is the Wulff shape centered at the origin with the same Lebesgue measure as $\Omega$. 
Remark 2.4. If $\Omega=\mathcal{W}_{R}$, by the simmetry of the problem, $T_{p}\left(\mathcal{W}_{R}\right)$ and the solution $u$ of (17) can be explicity calculated. We have:

$$
u_{\mathcal{W}}(x)=\frac{R^{q}-F^{o}(x)^{q}}{q N^{q-1}} \text { and } T_{p}\left(\mathcal{W}_{R}\right)=\frac{1}{N^{q-1}} \frac{\left|\mathcal{W}_{R}\right|}{N+q} R^{q}
$$

Remark 2.5. We point out that the lower bound in statement ii) of Theorem 1.I gives a stability type inequality for (22). Indeed we have

$$
0 \leqslant T_{p}\left(\mathcal{W}_{R}\right)-T_{p}(\Omega) \leqslant \frac{1}{8}\left(R^{2}-R_{F}^{2}(\Omega)\right)
$$

where $\left|\mathcal{W}_{R}\right|=|\Omega|$.

\section{AN ESTIMATE OF THE MAXIMUM OF THE TORSION FUNCTION}

In order to give a sharp upper bound for the maximum $M(\Omega)$ of the torsion function $u_{\Omega}$, we will take into account the following $\mathcal{P}$-function:

$$
\mathcal{P}(x)=\frac{p-1}{p} F^{p}\left(\nabla u_{\Omega}\right)+u_{\Omega}-M(\Omega),
$$

where $M(\Omega)=\max _{\Omega} u_{\Omega}$. The following result is proved in [CFV].

Proposition 3.1. Let $\Omega$ be a domain in $\mathbb{R}^{\mathrm{N}}, \mathrm{N} \geqslant 2$, and $\mathrm{u}_{\Omega} \in \mathrm{W}_{0}^{1, p}(\Omega)$ be a solution of (17). Set

$$
d_{i j}:=\frac{1}{F\left(\nabla u_{\Omega}\right)} \partial \xi_{i} \xi_{j}\left[\frac{F^{p}}{p}\right]\left(\nabla u_{\Omega}\right),
$$

Then it holds that

$$
\left(d_{i j} \mathcal{P}_{i}\right)_{j}-b_{k} \mathcal{P}_{k} \geqslant 0 \quad \text { in }\left\{\nabla u_{\Omega} \neq 0\right\}
$$

where

$$
b_{k}=\frac{p-2}{F^{3}\left(\nabla u_{\Omega}\right)} F_{\xi_{\ell}}\left(\nabla u_{\Omega}\right) \mathcal{P}_{x_{\ell}} F_{\xi_{k}}\left(\nabla u_{\Omega}\right)+\frac{2 p-3}{F^{2}\left(\nabla u_{\Omega}\right)}\left(\frac{F_{\xi_{k} \xi_{\ell}}\left(\nabla u_{\Omega}\right) \mathcal{P}_{x_{\ell}}}{p-1}-F_{\xi_{k}}\left(\nabla u_{\Omega}\right)\right)
$$

As a consequence of the previous result we get the following maximum principle for $\mathcal{P}$.

Theorem 3.2. Let $\Omega$ be a bounded $\mathrm{C}^{2}$ domain in $\mathbb{R}^{\mathrm{N}}, \mathrm{N} \geqslant 2$, with nonnegative anisotropic mean curvature $\mathcal{H}_{\mathrm{F}} \geqslant 0$ on $\partial \Omega$, and $\mathrm{u}_{\Omega}$ the torsion function. Then

$$
\frac{p-1}{p} F^{p}\left(\nabla u_{\Omega}\right)+u_{\Omega} \leqslant M(\Omega) \quad \text { in } \bar{\Omega},
$$

that is the function $\mathcal{P}$ achieves its maximum at the points $\mathrm{x}_{M} \in \Omega$ such that $\mathrm{u}_{\Omega}\left(\mathrm{x}_{M}\right)=M(\Omega)$.

Proof. Let us denote by $\mathrm{E}_{\mathrm{u}_{\Omega}}$ the set of the critical points of $\mathrm{u}_{\Omega}$, that is $\mathrm{E}_{\mathfrak{u}_{\Omega}}=\left\{x \in \bar{\Omega}: \nabla \mathrm{u}_{\Omega}(\mathrm{x})=\right.$ $0\}$. Being $\partial \Omega C^{2}$, by the Hopf Lemma (see for example [CT]), $E_{u_{\Omega}} \cap \partial \Omega=\emptyset$.

Applying Proposition 3.1, the function $\mathcal{P}$ verifies a maximum principle in the open set $\Omega \backslash E_{\mathfrak{u}_{\Omega}}$. Then we have

$$
\max _{\Omega \backslash \mathrm{E}_{\mathfrak{u}_{\Omega}}} \mathcal{P}=\max _{\partial\left(\Omega \backslash \mathrm{E}_{\mathfrak{u}_{\Omega}}\right)} \mathcal{P} .
$$

Hence one of the following three cases occur 
1. the maximum point of $\mathcal{P}$ is on $\partial \Omega$;

2. the maximum point of $\mathcal{P}$ is on $E_{u}$;

3. the function $\mathcal{P}$ is constant in $\bar{\Omega}$.

In order to prove the theorem we have to show that statement 1 cannot happen. Let us compute the derivative of $\mathcal{P}$ in the direction of the anisotropic normal $n_{F}$, in the sense of (13). Hence we get

$$
\begin{aligned}
& \frac{\partial \mathcal{P}}{\partial n_{\mathrm{F}}}=\frac{p-1}{p} \frac{\partial}{\partial n_{\mathrm{F}}}\left(-\frac{\partial u_{\Omega}}{\partial n_{\mathrm{F}}}\right)^{p}+\frac{\partial u_{\Omega}}{\partial n_{\mathrm{F}}}=-(p-1)\left(-\frac{\partial u}{\partial n_{\mathrm{F}}}\right)^{p-1} \frac{\partial^{2} u_{\Omega}}{\partial n_{\mathrm{F}}^{2}}+\frac{\partial u_{\Omega}}{\partial n_{\mathrm{F}}}= \\
&=-\mathrm{F}\left(\nabla \mathrm{u}_{\Omega}\right) \mathcal{Q}_{\mathrm{p}}[\mathrm{u}]-\mathrm{F}^{p-1}\left(\nabla \mathrm{u}_{\Omega}\right) \mathcal{H}_{\mathrm{F}}-\mathrm{F}\left(\nabla \mathrm{u}_{\Omega}\right)=-\mathrm{F}^{p-1}\left(\nabla \mathrm{u}_{\Omega}\right) \mathcal{H}_{\mathrm{F}},
\end{aligned}
$$

where last identity follows by (15). On the other hand, if a maximum point $\bar{x}$ of $\mathcal{P}$ is on $\partial \Omega$, by Hopf Lemma either $\mathcal{P}$ is constant in $\bar{\Omega}$, or $\frac{\partial \mathcal{P}}{\partial \mathfrak{n}_{\mathrm{F}}}(\bar{x})>0$. Hence being $\mathcal{H}_{\mathrm{F}} \geqslant 0$ we have a contradiction.

As a consequence of the previous result we get the following optimal estimate for the maximum of $\mathfrak{u}_{\Omega}$.

Theorem 3.3. Let $\Omega$ be a bounded convex domain in $\mathbb{R}^{\mathrm{N}}, \mathrm{N} \geqslant 2$, and $1<\mathrm{p}<+\infty$. It holds that

$$
\frac{\mathrm{R}_{\mathrm{F}}^{\mathrm{q}}(\Omega)}{\mathrm{qNq}-1} \leqslant M(\Omega) \leqslant \frac{\mathrm{R}_{\mathrm{F}}^{\mathrm{q}}(\Omega)}{\mathrm{q}} .
$$

Remark 3.4. In the next section we will show that the right-hand side inequality in (25) is optimal on a suitable sequence of thinning rectangles (see Proposition 4.4 and (36). We stress that, in general, the quotient $\frac{\mathrm{R}_{\mathrm{F}}^{\mathrm{q}}(\Omega)}{\mathrm{q} \mathrm{M}(\Omega)}$ approaches the value 1 also for different sequences of sets (see the example 4.7).

Proof. The left-hand side inequality of (25) follows by (21) and (23). Hence, let us prove the other inequality.

First of all, suppose that $\Omega$ is a $C^{2}$, strictly convex domain. Let $v$ be a direction in $\mathbb{R}^{\mathrm{N}}$. By Theorem 3.2 and property (7) we have

$$
\frac{\mathrm{d} u_{\Omega}}{\mathrm{d} v}=\langle\nabla u, v\rangle \leqslant \mathrm{F}\left(\nabla \mathrm{u}_{\Omega}\right) \mathrm{F}^{\mathrm{o}}(v) \leqslant\left[\frac{\mathrm{p}}{\mathrm{p}-1}\left(\mathrm{M}(\Omega)-\mathrm{u}_{\Omega}\right)\right]^{\frac{1}{\mathrm{p}}} \mathrm{F}^{\mathrm{o}}(v),
$$

where $M(\Omega)$ is the maximum of $u_{\Omega}$ in $\bar{\Omega}$. Let us denote by $x_{M}$ the point of $\Omega$ such that $M(\Omega)=u_{\Omega}\left(x_{M}\right)$, by $\bar{x} \in \partial \Omega$ such that $F^{o}\left(x_{M}-\bar{x}\right)=d_{F}\left(x_{M}\right)$ and by $v$ the direction of the straight line joining the points $x_{m}$ and $\bar{x}$. Then by (26) we get

$$
\int_{u(\bar{x})}^{M(\Omega)} \frac{1}{\left(M(\Omega)-u_{\Omega}\right)^{\frac{1}{p}}} d u \leqslant\left(\frac{p}{p-1}\right)^{\frac{1}{p}} F^{o}(v)\left|\bar{x}-x_{M}\right|=\left(\frac{p}{p-1}\right)^{\frac{1}{p}} F^{o}\left(\bar{x}-x_{M}\right) .
$$

Being $F^{O}\left(\bar{x}-x_{M}\right) \leqslant R_{F}(\Omega)$, we get

$$
\left(\frac{p}{p-1}\right) M(\Omega)^{\frac{p-1}{p}} \leqslant\left(\frac{p}{p-1}\right)^{\frac{1}{p}} R_{F}(\Omega),
$$

which gives the estimate (25) for smooth convex domains. To prove the estimate in the case of a general convex body $\Omega$, we proceed by approximation. It is well-known (see for example [BF]) that a convex body $\Omega$ can be approximated in the Hausdorff distance by an increasing sequence of smooth strictly convex bodies $\Omega_{n} \subseteq \Omega$. Clearly, $R_{F}\left(\Omega_{n}\right) \subsetneq R_{F}(\Omega)$. 
Let $u_{n} \geqslant 0$ be the torsion function in $\Omega_{n}$. In order to conclude the proof we have to show that $M\left(\Omega_{n}\right) \rightarrow M(\Omega)$ as $n \rightarrow \infty$. We first observe that by (25),

$$
M\left(\Omega_{n}\right) \leqslant \frac{R_{F}^{q}\left(\Omega_{n}\right)}{q} \leqslant \frac{R_{F}^{q}(\Omega)}{q}
$$

hence $u_{n}$ are bounded in $L^{\infty}\left(\Omega_{n}\right)$. Furthermore, applying Theorem 3.2 in $\Omega_{n}$ we have

$$
\frac{p-1}{p} F^{p}\left(\nabla u_{n}\right)+u_{n} \leqslant M\left(\Omega_{n}\right) \quad \text { in } \bar{\Omega}_{n} .
$$

Then by property (5)

$$
\left|\nabla u_{n}\right| \leqslant C \quad \text { in } \bar{\Omega}_{n} .
$$

Hence by (27) and (28), using Ascoli-Arzelà theorem we get that $\mathfrak{u}_{n} \rightarrow \mathfrak{u}_{\Omega}$ uniformly in $\Omega$ and this allows to pass to the limit in (27) and the proof is completed.

Remark 3.5. We point out that if we take $\Omega$ smooth, the thesis of Theorem 3.3 holds if we assume only that the anisotropic mean curvature of $\Omega$ is nonnegative.

\section{PROOF OF THEOREM 1.1}

We split the proof in various theorems. We first prove the lower bound for $\Psi(\Omega)$ in ii).

Theorem 4.1. If $\Omega \in \mathbb{R}^{N}$ is a convex bounded domain, $N \geqslant 2$, and $1<p<+\infty$, then

$$
\frac{\mathrm{T}_{\mathrm{p}}(\Omega)}{|\Omega|} \geqslant \frac{1}{\mathrm{Nq}-1} \frac{1}{\mathrm{~N}+\mathrm{q}} \mathrm{R}_{\mathrm{F}}(\Omega)^{\mathrm{q}}
$$

where $R_{\mathrm{F}}(\Omega)$ is the anisotropic inradius of $\Omega$ defined in (16). Moreover the equality holds when $\Omega$ is a Wulff shape.

Proof. Let us assume first that $\Omega$ is a strictly convex domain and then we remove this assumption with a proof that follows by approximation as in Theorem 3.3. Let us consider as test function into (19) the following

$$
\varphi(x)=\frac{1-\mathcal{K}^{\mathrm{o}}(x)^{\mathrm{q}}}{\mathrm{qNq}^{-1}}
$$

where $\mathcal{K}^{\circ}$ is the support function of the polar set of $\Omega$, defined in (8). Then $\Omega=\left\{\mathcal{K}^{\mathrm{o}}<1\right\}$. By (23), we observe that when $\Omega=\mathcal{W}$ then $\varphi$ is exactly the torsion function of the Wulff shape.

We start computing

$$
\begin{aligned}
\int_{\Omega} \mathcal{K}^{\mathrm{o}}(\mathrm{x})^{\mathrm{q}} \mathrm{d} x & =\int_{0}^{1} \int_{\mathcal{K}^{\mathrm{o}=\mathrm{t}}} \frac{\mathrm{t}^{\mathrm{q}}}{\left|\nabla \mathcal{K}^{\mathrm{o}}(\mathrm{x})\right|} \mathrm{d} \mathcal{H}^{\mathrm{N}-1} \mathrm{dt}=\int_{0}^{1} \mathrm{t}^{\mathrm{q}} \int_{\mathcal{K}^{\mathrm{o}}=\mathrm{t}} \frac{\mathcal{K}\left(\nabla \mathcal{K}^{\mathrm{o}}(\mathrm{x})\right)}{\left|\nabla \mathcal{K}^{\mathrm{o}}\right|} \mathrm{d} \mathcal{H}^{\mathrm{N}-1} \mathrm{dt}, \\
& =\int_{0}^{1} \mathrm{t}^{\mathrm{q}+\mathrm{N}-1} \int_{\partial \Omega} \mathcal{K}\left(v_{\Omega}(x)\right) \mathrm{d} \mathcal{H}^{\mathrm{N}-1} \mathrm{dt}=\frac{1}{\mathrm{~N}+\mathrm{q}} \int_{\partial \Omega} x \cdot v_{\Omega}(x) \mathrm{d} \mathcal{H}^{\mathrm{N}-1} \\
& =\frac{1}{\mathrm{~N}+\mathrm{q}} \int_{\Omega} \operatorname{div} x \mathrm{~d} x=\frac{\mathrm{N}|\Omega|}{\mathrm{N}+\mathrm{q}}
\end{aligned}
$$

where $\mathcal{K}=\left(\mathcal{K}^{\mathrm{o}}\right)^{\mathrm{o}}$. Then we have

$$
\left(\int_{\Omega} \varphi \mathrm{d} x\right)^{p}=\frac{|\Omega|^{p}}{N^{q}(N+q)^{p}}=\left(\frac{|\Omega|}{N^{q-1}(N+q)}\right)^{p} .
$$


Let us now compute

$$
\begin{aligned}
\int_{\Omega} F^{p}(\nabla \varphi) d x & =\frac{1}{q^{p} N^{q}} \int_{\Omega} F^{p}\left(\nabla\left(\mathcal{K}^{o}(x)^{q}\right)\right) d x=\frac{1}{N^{q}} \int_{0}^{1} t^{p(q-1)} \int_{\mathcal{K}^{o}=t} \frac{F^{p}\left(\nabla \mathcal{K}^{o}\right)}{\left|\nabla \mathcal{K}^{o}\right|} d \mathcal{H}^{N-1} d t \\
& =\frac{1}{N^{q}} \int_{0}^{1} t^{q+N-1} \int_{\partial \Omega}\left|\nabla \mathcal{K}^{o}\right|^{p-1} F^{p}\left(v_{\Omega}\right) d \mathcal{H}^{N-1} d t \\
& =\frac{1}{N^{q}} \int_{0}^{1} t^{q+N-1} \int_{\partial \Omega} \frac{F^{p}\left(v_{\Omega}\right)}{\mathcal{K}^{p-1}\left(v_{\Omega}\right)} d \mathcal{H}^{N-1} d t
\end{aligned}
$$

where last equality follows by the identity $\mathcal{K}\left(\nabla \mathcal{K}^{\mathrm{o}}(x)\right)=1$. Being $\mathcal{W}_{\mathrm{R}_{\mathrm{F}}(\Omega)} \subseteq \Omega$, it follows that $\mathcal{K}(x) \geqslant R_{F}(\Omega) F(x)$, so we have

$$
\begin{aligned}
\int_{\Omega} \mathrm{F}^{\mathrm{p}}(\nabla \varphi) \mathrm{d} x \leqslant \frac{1}{(\mathrm{~N}+\mathrm{q}) \mathrm{N}^{\mathrm{q}}} \cdot \frac{1}{\mathrm{R}_{\mathrm{F}}(\Omega)^{\mathrm{p}}} \int_{\partial \Omega} \mathcal{K}\left(v_{\Omega}(x)\right) \mathrm{d} \mathcal{H}^{\mathrm{N}-1}= & \\
= & \frac{1}{(\mathrm{~N}+\mathrm{q}) \mathrm{Nq}-1} \cdot \frac{|\Omega|}{\mathrm{R}_{\mathrm{F}}(\Omega)^{\mathrm{p}}}
\end{aligned}
$$

Joining together (30) and (31), we have the thesis.

Now we prove the validity of (29) without the assumption on the strict convexity of the domain $\Omega$. As in the proof of Theorem 3.3, let $\Omega_{n}$ be a sequence of smooth strictly convex bodies such that $\Omega_{n} \rightarrow \Omega$. Such a convergence ensures that, as $n \rightarrow \infty$,

$$
\left|\Omega_{n}\right| \rightarrow|\Omega| \quad \text { and } \quad R_{F}\left(\Omega_{n}\right) \rightarrow R_{F}(\Omega) .
$$

By (20), it follows that

$$
\mathrm{T}_{\mathrm{p}}(\Omega) \geqslant \mathrm{T}_{\mathrm{p}}\left(\Omega_{\mathrm{n}}\right),
$$

and by applying (29) to each $\Omega_{n}$, we find

$$
\mathrm{T}_{p}(\Omega) \geqslant \frac{\left|\Omega_{n}\right|}{\mathrm{N}^{\mathrm{q}-1}(\mathrm{~N}+\mathrm{q})} \mathrm{R}_{\mathrm{F}}\left(\Omega_{\mathrm{n}}\right)^{\mathrm{q}},
$$

which, combined with (32), gives the desired result. Finally we stress that if $\Omega$ is a Wulff shape, the equality case follows from Remark 2.4. On the other hand, if the equality holds in (29), then equality must hold in (3I), and then $\mathcal{K}(x)=R_{F}(\Omega) F(x)$, which implies $\Omega=\mathcal{W}_{R_{F}}$.

Let us consider the functional

$$
\Phi(\Omega)=\frac{\mathrm{T}_{\mathrm{p}}(\Omega)}{|\Omega| M(\Omega)} .
$$

As consequence of theorems 4.1 and 3.3, we can prove the following estimates for (33) which is statement i) of Theorem 1.1.

Theorem 4.2. For any bounded convex domain $\Omega \subset \mathbb{R}^{N}, N \geqslant 2,1<p<+\infty$ it holds that

$$
\frac{\mathrm{q}}{\mathrm{Nq}^{-1}(\mathrm{~N}+\mathrm{q})} \leqslant \Phi(\Omega) \leqslant \frac{\mathrm{q}}{\mathrm{q}+1}
$$

Proof. We first prove the lower bound for the functional Ф. By (29) and (25) we have

$$
\Phi(\Omega)=\frac{T_{p}(\Omega)}{|\Omega| M(\Omega)} \geqslant \frac{q}{N^{q-1}(N+q)},
$$

which gives the lower bound in (34). 
In order to prove the inequality in the right-hand side in (34), by Theorem 3.2 we have

$$
\frac{p-1}{p} F^{p}\left(\nabla u_{\Omega}\right)+u_{\Omega} \leqslant M(\Omega) \text { in } \bar{\Omega} .
$$

Integrating in both sides and recalling (18), we get

$$
\left(\frac{p-1}{p}+1\right) T_{p}(\Omega) \leqslant M(\Omega)|\Omega|
$$

which implies the upper bound in (34).

In the following last result we prove the upper bound in statement ii) of Theorem 1.1, which follows immediately by the preceding results. We stress that in the anisotropic setting, the case $p=2$ was previously considered in [BGM] with a completely different proof.

Theorem 4.3. Let $\Omega \in \mathbb{R}^{N}$ be a bounded convex domain, $N \geqslant 2,1<p<+\infty$. It holds that

$$
\frac{T_{p}(\Omega)}{|\Omega|} \leqslant \frac{R_{F}(\Omega)^{q}}{q+1} .
$$

Proof. By the right-hand side inequality in (34), and (25), we have

$$
\frac{T_{p}(\Omega)}{|\Omega|} \leqslant \frac{q}{q+1} M(\Omega) \leqslant \frac{R_{F}^{q}(\Omega)}{q+1} .
$$

The final part of the section is devoted to prove the optimality of (35). As a consequence, by (36) this will give the optimality of the right-hand side inequality of (34), and of (25).

Proposition 4.4. Let $\Omega_{\varepsilon}$ be the $N$-rectangle $]-\varepsilon, \varepsilon[\times]-a_{2}, a_{2}[\times \ldots \times]-a_{N}, a_{N}[$, and suppose that $\mathrm{R}_{\mathrm{F}}(\Omega)=\varepsilon \mathrm{F}^{\mathrm{O}}\left(\mathrm{e}_{1}\right)$. Then

$$
\lim _{\varepsilon \rightarrow 0^{+}} \frac{T_{p}\left(\Omega_{\varepsilon}\right)}{\left(R_{F}\left(\Omega_{\varepsilon}\right)\right)^{q}\left|\Omega_{\varepsilon}\right|}=\frac{1}{q+1}
$$

The hypothesis $R_{F}(\Omega)=\varepsilon^{F}\left(e_{1}\right)$ is not restrictive, in the sense that if it is not true we can choose a rotated N-rectangle where $R_{F}(\Omega)=\varepsilon^{\circ}(v)$ for some direction $v$, and use the remark below.

Remark 4.5. If $A \in S O(N)$ is a rotation matrix, then, denoting by $F_{A}(\xi)=F(A \xi)$, it holds that

$$
\left(F_{A}\right)^{o}(\xi)=\left(F^{o}\right)_{A}(\xi) \text {, and then } R_{F_{A} T}(A \Omega)=R_{F}(\Omega)
$$

(see [DGP] for the details). Hence, emphasizing the dependence on $F$ by denoting $T_{p}(\Omega)=$ $T_{p, F}(\Omega)$, we have

$$
\begin{aligned}
\mathrm{T}_{\mathrm{p}, \mathrm{F}}(\Omega) & =\max _{\varphi \in \mathrm{W}_{0}^{1, p}(\Omega)} \frac{\left(\int_{\Omega}|\varphi(x)| \mathrm{d} x\right)^{\mathrm{p}}}{\int_{\Omega} \mathrm{F}^{\mathrm{p}}(\nabla \varphi(x)) \mathrm{d} x}=\max _{\varphi \in W_{0}^{1, p}(\Omega)} \frac{\left(\int_{A \Omega}\left|\varphi\left(A^{\top} x\right)\right| \mathrm{d} x\right)^{p}}{\int_{A \Omega} \mathrm{F}_{A^{\mathrm{T}}}^{\mathrm{p}}\left(\nabla \varphi\left(A^{\top} x\right)\right) \mathrm{d} x} \\
& =\mathrm{T}_{\mathrm{p}, \mathrm{F}_{\mathrm{A}} \mathrm{T}}(A \Omega) \geqslant \frac{|\Omega|}{\mathrm{q}+1} \mathrm{R}_{\mathrm{F}_{\mathrm{A}} \mathrm{T}}(A \Omega)^{\mathrm{q}}=\frac{|\Omega|}{\mathrm{q}+1} \mathrm{R}_{\mathrm{F}}(\Omega)^{\mathrm{q}} .
\end{aligned}
$$


Proof of Proposition 4.4. First of all, we observe that

$$
\mathrm{F}^{\mathrm{o}}\left(\mathrm{e}_{1}\right)=\frac{1}{\mathrm{~F}\left(e_{1}\right)}
$$

Indeed, being $R_{F}(\Omega)=\varepsilon F^{o}\left(e_{1}\right)$, it holds that

$$
v_{\Omega}\left(\varepsilon e_{1}\right)=e_{1}=\frac{F_{\xi}^{o}\left(e_{1}\right)}{\left|F_{\xi}^{o}\left(e_{1}\right)\right|^{\prime}},
$$

where $v_{\Omega}\left(\varepsilon e_{1}\right)$ is the Euclidean outer normal vector to $\partial \Omega$. Hence by (10) and (9), we have

$$
\mathrm{F}\left(e_{1}\right)=\frac{1}{\left|\mathrm{~F}_{\xi}^{\mathrm{o}}\left(e_{1}\right)\right|}=\frac{1}{\mathrm{~F}^{\mathrm{o}}\left(e_{1}\right)}
$$

where last equality follows by $F^{o}\left(e_{1}\right)=F_{\xi}^{o}\left(e_{1}\right) \cdot e_{1}=\left|F_{\xi}^{o}\left(e_{1}\right)\right|$.

Let $\Omega_{\varepsilon}=C_{\varepsilon} \cup D_{\varepsilon}$, where $\left.C_{\varepsilon}=\right]-\varepsilon, \varepsilon[\times] a_{2}+\varepsilon, a_{2}-\varepsilon[\times \ldots \times]-a_{N}+\varepsilon, a_{N}-\varepsilon\left[\right.$, and $D_{\varepsilon}=$ $\Omega_{\varepsilon} \backslash C_{\varepsilon}$. Setting $x=\left(x_{1}, z\right)$ with $z \in \mathbb{R}^{N-1}$ and $a=\left(a_{2}, \ldots, a_{N}\right)$, we consider the function $\varphi_{\varepsilon}$ defined by

$$
\begin{cases}\varphi_{\varepsilon}\left(x_{1}, z\right)=\frac{\varepsilon^{q}-x_{1}^{q}}{q} & \text { in } C_{\varepsilon} \\ \varphi_{\varepsilon}\left(x_{1}, z\right)=\min \{|a-z|,|-a-z|\} \frac{\varepsilon^{q}-x_{1}^{q}}{q \varepsilon} & \text { in } D_{\varepsilon} .\end{cases}
$$

We can estimate the anisotropic p-torsional rigidity by using $\varphi_{\varepsilon}$ as test function. We have:

$$
\mathrm{T}_{p}\left(\Omega_{\varepsilon}\right)^{p-1} \geqslant \frac{\left(\int_{\Omega_{\varepsilon}} \varphi_{\varepsilon}\right)^{p}}{\int_{\Omega_{\varepsilon}} F^{p}\left(\nabla \varphi_{\varepsilon}\right) d x}=\frac{\left(\int_{C_{\varepsilon}} \varphi_{\varepsilon}+\int_{D_{\varepsilon}} \varphi_{\varepsilon} d x\right)^{p}}{\int_{C_{\varepsilon}} F^{p}\left(\nabla \varphi_{\varepsilon}\right) d x+\int_{D_{\varepsilon}} F^{p}\left(\nabla \varphi_{\varepsilon}\right) d x} .
$$

We now compute

$$
\int_{C_{\varepsilon}} \varphi_{\varepsilon} d x=\int_{C_{\varepsilon}} \frac{\varepsilon^{q}-x_{1}^{q}}{q} d x=\frac{\left|C_{\varepsilon}\right| \varepsilon^{q}}{q+1}
$$

and

$$
\int_{C_{\varepsilon}} F^{p}\left(\nabla \varphi_{\varepsilon}\right) d x=F^{p}\left(e_{1}\right) \int_{C_{\varepsilon}} x_{1}^{q} d x=F^{p}\left(e_{1}\right) \frac{\left|C_{\varepsilon}\right| \varepsilon^{q}}{q+1} .
$$

We notice that both $\int_{D_{\varepsilon}} \varphi_{\varepsilon} d x$ and $\int_{D_{\varepsilon}} F^{p}\left(\nabla \varphi_{\varepsilon}\right) d x$ are negligible, since they go to zero as $\varepsilon^{\mathrm{N}+\mathrm{q}-1}$. By recalling that

$$
\left(R_{F}(\Omega)\right)^{q}=\varepsilon^{q} F^{o}\left(e_{1}\right)^{q}=\frac{\varepsilon^{q}}{F\left(e_{1}\right)^{q}},
$$

we have

$$
\frac{1}{q+1} \geqslant \lim _{\varepsilon \rightarrow 0} \frac{T_{p}\left(\Omega_{\varepsilon}\right)}{\left(R_{F}\left(\Omega_{\varepsilon}\right)\right)^{q}\left|\Omega_{\varepsilon}\right|} \geqslant \frac{1}{q+1}
$$

which concludes the proof. 
Remark 4.6. We believe that the lower bound of $\Phi(\Omega)$ in (34) is not optimal. Actually, in the Euclidean setting, with $p=2$ our bound improves the analogous result of [HLP]:

$$
\Phi(\Omega) \geqslant \frac{2}{N(N+2)}>\frac{1}{(N+1)^{2}}
$$

Moreover in [HLP] the authors conjecture that for $\mathrm{F}=\mathcal{E}, \mathrm{p}=2$ and $\mathrm{N}=2$ it holds

$$
\Phi(\Omega) \geqslant \frac{1}{3}
$$

and

$$
\Phi\left(\Omega_{\mathrm{n}}\right) \rightarrow \frac{1}{3}, \quad \text { as } \mathrm{n} \rightarrow \infty,
$$

where $\Omega_{n}$ is a sequence of isosceles triangles degenerating to a segment.

In the following example, for $F=\mathcal{E}, N=2$ and $p=2$, we find a sequence of degenerating triangles $\Omega_{\mathfrak{n}}$ such that (37) holds.

\section{Example 4.7. Let}

$$
N=2, \quad F(\xi)=\sqrt{\xi_{1}^{2}+\xi_{2}^{2}}, \quad p=2 .
$$

We want to show that there exists a sequence of thinning isosceles triangles $\tau_{a}$ of the plane such that

$$
\Phi\left(\tau_{a}\right)=\frac{T_{2}\left(\tau_{a}\right)}{\left|\tau_{a}\right| M\left(\tau_{a}\right)} \rightarrow \frac{1}{3} \quad \text { as } \quad a \rightarrow 0,
$$

where $T_{2}\left(\tau_{a}\right)$ is the torsional rigidity of $\tau_{a}, M\left(\tau_{a}\right)$ is the maximum of the torsion function in $\tau_{a}$ and $\left|\tau_{a}\right|$ is the area of the triangle.

First of all, we recall that by a result contained in [FGL], for any sequence of isosceles triangles $\tau_{n}$ such that the ratio $\frac{R\left(\tau_{n}\right)}{w\left(\tau_{n}\right)} \rightarrow 0$, where $w\left(\tau_{n}\right)$ is the width of $\tau_{n}$, then

$$
\lim _{n \rightarrow \infty} \frac{T_{2}\left(\tau_{n}\right)}{\left|\tau_{n}\right|} \frac{P^{2}\left(\tau_{n}\right)}{\left|\tau_{n}\right|^{2}}=\frac{2}{3}
$$

Hence, recalling that in a triangle it holds that $R\left(\tau_{n}\right)=\frac{2\left|\tau_{n}\right|}{P\left(\tau_{n}\right)}$ then

$$
\Phi\left(\tau_{n}\right)=\frac{T_{2}\left(\tau_{n}\right)}{\left|\tau_{n}\right|} \frac{P^{2}\left(\tau_{n}\right)}{\left|\tau_{n}\right|^{2}} \frac{R^{2}\left(\tau_{n}\right)}{4 M\left(\tau_{n}\right)}
$$

the result is proved if we find a sequence of triangles with vanishing ratio $R\left(\tau_{n}\right) / w\left(\tau_{n}\right)$ and such that $\frac{R^{2}\left(\tau_{n}\right)}{2 M\left(\tau_{n}\right)}$ tends to 1 .

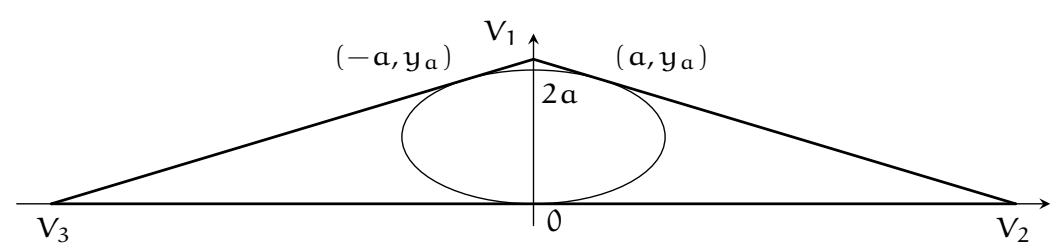

Figure 1

To this aim, let

$$
\mathcal{E}_{a}=\left\{(x, y) \in \mathbb{R}^{2}: \frac{x^{2}}{1-a^{2}}+\frac{(y-a)^{2}}{a^{2}}=1\right\},
$$


and consider a point $\left(a, y_{a}\right)$, with $y_{a}=a+a \sqrt{\frac{1-2 a^{2}}{1-a^{2}}}$. Let $\tau_{a}$ be the isosceles triangle constructed with one side on the $x$-axis and with each side tangent to the ellipse at the points $(0,0),\left(a, y_{a}\right),\left(-a, y_{a}\right)$, as in Figure 1 .

The vertices of the triangle are:

$$
v_{1}=\left(0, y_{a}+\frac{a^{4}}{\left(1-a^{2}\right)\left(y_{a}-a\right)}\right), \quad v_{2}=\left(a+\frac{y_{a}\left(y_{a}-a\right)}{a^{3}}\left(1-a^{2}\right), 0\right), \quad v_{3}=-v_{2}
$$

Let us observe that $V_{1} \rightarrow(0,0)$ as $a \rightarrow 0$, while the first coordinate of $V_{2}$ diverges.

Then, denoting by $A\left(\tau_{a}\right)$ and $P\left(\tau_{a}\right)$ respectively the area and the perimeter of $\tau_{a}$, and by

$$
h=y_{a}+\frac{a^{4}}{\left(1-a^{2}\right)\left(y_{a}-a\right)}, \quad \frac{b}{2}=a+\frac{y_{a}\left(y_{a}-a\right)}{a^{3}}\left(1-a^{2}\right),
$$

we have:

$$
R\left(\tau_{a}\right)=\frac{2\left|\tau_{a}\right|}{P\left(\tau_{a}\right)}=\frac{b h}{b+\sqrt{2 h^{2}+b^{2}}} .
$$

Now, being $\mathcal{E}_{\mathrm{a}} \subset \tau_{\mathrm{a}}$, by the comparison principle and (25) it holds that

$$
M\left(\varepsilon_{a}\right) \leqslant M\left(\tau_{a}\right) \leqslant \frac{R^{2}\left(\tau_{a}\right)}{2}, \quad M\left(\varepsilon_{a}\right)=\frac{a^{2}\left(1-a^{2}\right)}{2},
$$

where the maximum of the torsion function on $\varepsilon_{a}$ follows by a direct computation. Then, being $h=2 a+o\left(a^{2}\right)$ and $b \rightarrow+\infty$ as $a \rightarrow 0$, we have

$$
\begin{array}{r}
1 \leqslant \frac{R\left(\tau_{a}\right)^{2}}{2 M\left(\varepsilon_{a}\right)}=\left(\frac{b}{b+\sqrt{2 h^{2}+b^{2}}}\right)^{2} \frac{h^{2}}{a^{2}\left(1-a^{2}\right)}=\left(\frac{1}{1+\sqrt{2 \frac{h^{2}}{b^{2}}+1}}\right)^{2} \cdot \frac{h^{2}}{a^{2}\left(1-a^{2}\right)} \\
\text { as } a \rightarrow 0
\end{array}
$$

and $(38)$ is proved.

We explicitly observe that, from the above computations, it holds

$$
\frac{R^{2}\left(\varepsilon_{a}\right)}{2 M\left(\varepsilon_{a}\right)} \rightarrow 1 \quad \text { as } a \rightarrow 0 .
$$

\section{ACNOWLEDGEMENTS}

This work has been partially supported by the FIRB 2013 project "Geometrical and qualitative aspects of PDE's" and by GNAMPA of INdAM.

\section{REFERENCES}

[AFLT] Alvino A., Ferone V., Lions P.-L., Trombetti G., Convex symmetrization and applications, Ann. Inst. H. Poincaré Anal. Non Linéaire, 14(2):275-293, 1997. 6

[vB] van den Berg M.: Estimates for the Torsion Function and Sobolev Constants, Potential Anal. 36, 607-616 (2012). 3 
[vBBV] van den Berg M., Buttazzo G., Velichkov B.: Optimization problems involving the first Dirichlet eigenvalue and the torsional rigidity, New trends in shape optimization, Internat. Ser. Numer. Math. 166, pp. 19-41. Birkhäuser/Springer (2015). 3

[vBFNT] van den Berg M., Ferone V., Nitsch C., Trombetti C.,On Pólya's Inequality for Torsional Rigidity and First Dirichlet Eigenvalue, Integr. Equ. Oper. Theory 86, 579-60o (2016). 3

[BF] Bonnesen T., Fenchel W. Theorie der Konvexen Körper. Springer, Berlin, 1934. 8

[BFr] Borisov D., Freitas P., Asymptotics for the Expected Lifetime of Brownian Motion on Thin Domains in $\mathbb{R}^{n}$, J. Theor. Probab. 26:284-309 (2013). 3

[BGM] Buttazzo G., Guarino Lo Bianco S., Marini M., Sharp estimates for the anisotropic torsional rigidity and the principal frequency, J. Math. Anal. Appl. to appear, DOI:10.1016/j.jmaa.2017.03.055. 2, 11

[CFV] Cozzi M., Farina A., Valdinoci E., Gradient Bounds and Rigidity Results for Singular, Degenerate, Anisotropic Partial Differential Equations, Comm. Math. Phys., 189-214 (2014). 7

[CM] Crasta G., Malusa A., The distance function from the boundary in a Minkowski space. Trans. Amer. Math. Soc., 359(12):5725-5759 (2007). 5

[CT] Cuesta M., Takáč P., A strong comparison principle for positive solutions of degenerate elliptic equations. Diff. Int. Eq. 13 (2000): 721-746. 7

[DG1] Della Pietra F., Gavitone N., Sharp bounds for the first eigenvalue and the torsional rigidity related to some anisotropic operators, Math. Nachr. 287, 194-209 (2014). 3, 6

[DGP] Della Pietra F., Gavitone N., Piscitelli G., A sharp weighted anisotropic Poincaré inequality for convex domains, preprint, 2017. II

[FGL] Fragalà I., Gazzola F., Lamboley J., Sharp Bounds for the p-Torsion of Convex Planar Domains, Geometric Properties for Parabolic and Elliptic PDE's Springer INdAM Series Volume 2, 97-115 (2013). 2, 3, 13

[HLP] Henrot A., Lucardesi I., Philippin G., On two functionals involving the maximum of the torsion function, preprint. 2, 3, 13

[LU] Ladyzhenskaya O. A., Ural'tseva N. N. Linear and quasilinear elliptic equations. Translated from the Russian by Scripta Technica, Inc. Translation editor: Leon Ehrenpreis. Academic Press, New York, 1968. 6

[M] Makai E., On the principal frequency of a membrane and the torsional rigidity of a beam, Studies in mathematical analysis and related topics, 227-231, Stanford Univ. Press, Stanford, Calif. 1962. 2

[P] Payne L.E., Bounds for the maximum Stress in the St-Venant problem, Indian Journal of Mechanics and Mathematics, special issue in honor of B. Sen, part 1, 51-59 (1968) 3

[PZ] Pólya G., Szegő G.: Isoperimetric inequalities in Mathematical physics. Ann. of Math. Stud. 27, Princeton University Press, Princeton 1951. 2

[To] Tolksdorf P., Regularity for a more general class of quasilinear elliptic equations, J. Diff. Eq., 51(1):126-150 (1984). 6

[S] Sperb R., Maximum principles and applications, Academic Press, 1981. 3

[X] Xia C., On a class of anisotropic problems, Ph.D. Thesis. 5 